\title{
Desenvolvimento de um aplicativo para atendimento médico de pessoas que vivem com HIV/AIDS
}

\author{
Developing an application for medical care for people living with HIV/AIDS \\ Desarrollo de uma aplicación para atención médica de personas que viven com VIH/SIDA
}

Herbert Paulino Cordeiro ${ }^{1 *}$, Rafael de Azevedo Silva², Lorena Fecury Tavares², Cléa Nazaré Carneiro Bichara ${ }^{3}$.

\begin{abstract}
RESUMO
Objetivo: Desenvolver um aplicativo para dispositivos móveis com a finalidade de auxiliar as condutas durante o atendimento de pessoas vivendo com HIV/AIDS de acordo com o Protocolo Clínico de Diretrizes Terapêuticas para manejo da infecção pelo HIV em adultos. Método: Foram utilizadas ferramentas Android Studio na linguagem Java, emulador SDK Android e a ferramenta SQLite, desenvolvendo uma plataforma com informações divididas em seis eixos: exames na primeira consulta médica, início da terapia antirretroviral, profilaxias primárias, quando encaminhar ao especialista, imunizações e interações medicamentosas entre antiretrovirais e outros medicamentos. Resultados: A utilização de aplicativos em smartphone e tablets se tornou, com o desenvolvimento tecnológico, uma ferramenta de consulta e atualização do profissional médico. Assim, devido a diversas dificuldades encontradas na rede de atenção básica, o aplicativo desenvolvido é uma ferramenta que pode ser utilizada na atenção básica por profissionais da área médica, de modo off-line (não necessitando de internet) contendo o manejo preconizado pelo Ministério da Saúde de pacientes HIV/AIDS e compatível com a maioria dos smartphones e tablets que utilizem sistema Android. Conclusão: O aplicativo App HIV desenvolvido é de grande utilidade em orientar o médico da atenção básica para manejo de pacientes com HIV/AIDS em consultas iniciais.
\end{abstract}

Palavras-chave: Comportamentos Relacionados com a Saúde; Síndrome de Imunodeficiência Adquirida; Educação Continuada; HIV; Aplicativos Móveis.

\begin{abstract}
Objective: To develop an application for mobile devices in order to assist the conduct of care of people living with HIV / AIDS in accordance with the Clinical Protocol of Therapeutic Guidelines for the management of HIV infection in adults. Method: Android Studio tools were used in Java language, Android SDK emulator and SQLite tool, developing a platform with information divided into six axes: examinations at the first medical appointment, initiation of antiretroviral therapy, primary prophylaxis, referral to the specialist, immunizations and drug interactions between antiretrovirals and other medicinal products. Results: The use of smartphone and tablet applications has become, with technological development, a tool for consultation and updating of the medical professional. Thus, due to several difficulties found in the basic care network, the developed application is a tool that can be used in the basic care by medical professionals, offline (not requiring internet) containing the management recommended by the Ministry of Health Health of HIV / AIDS patients and compatible with most smartphones and tablets that use Android system. Conclusion: The developed HIV App application is very useful in guiding the primary care physician to manage patients with HIV / AIDS in initial consultations.
\end{abstract}

Keywords: Health Behavior; Acquired Immunodeficiency Syndrome; Education, Continuing; HIV; Mobile Aplications.

\footnotetext{
${ }^{1}$ Universidade Estadual do Pará (UEPA) e Centro Universitário Metropolitano da Amazônia (UNIFAMAZ) Belém, Pará. *E-mail: hpcordeiro@hotmail.com

${ }^{2}$ Centro Universitário Metropolitano da Amazônia (UNIFAMAZ) - Belém, Pará.

${ }_{3}^{3}$ Universidade do Estado do Pará (UEPA) - Belém, Pará.
} 


\section{RESUMEN}

Objetivo: Desarrollar una aplicación para dispositivos móviles con el fin de auxiliar las conductas durante la atención de personas viviendo con VIH / SIDA de acuerdo con el Protocolo Clínico de Directrices Terapéuticas para el manejo de la infección por VIH en adultos. Método: Se utilizaron herramientas Android Studio en el lenguaje Java, emulador SDK Android y la herramienta SQLite, desarrollando una plataforma con informaciones divididas en seis ejes: exámenes en la primera consulta médica, inicio de la terapia antirretroviral, profilaxias primarias, cuando encaminar al especialista, inmunizaciones y las interacciones medicamentosas entre antirretrovirales y otros medicamentos. Resultados: El uso de aplicaciones en smartphone y tablets se ha convertido, con el desarrollo tecnológico, una herramienta de consulta y actualización del profesional médico. Por lo tanto, debido a diversas dificultades encontradas en la red de atención básica, la aplicación desarrollada es una herramienta que puede ser utilizada en la atención básica por profesionales del área médica, de modo off-line (no necesitando de internet) conteniendo el manejo preconizado por el Ministerio de Salud de pacientes VIH / SIDA y compatible con la mayoría de los smartphones y tabletas que utilizan sistema Android. Conclusión: La aplicación App HIV desarrollado es de gran utilidad en orientar al médico de la atención básica para el manejo de pacientes con VIH / SIDA en consultas iniciales.

Palavras-clave: Conductas Relacionadas con la Salud; Síndrome de Imunodeficiencia Adquirida; Educación Continua; VIH; Aplicaciones Móveis.

\section{INTRODUÇÃO}

O número de pessoas que vivem com HIV/AIDS (Vírus da Imunodeficiência Humana/Síndrome da Imunodeficiência Adquirida) é uma parcela significativa da população no Brasil, haja vista de 2007 a 2017, houve 247.795 casos notificados, sendo o pretexto de nos últimos anos o Ministério da Saúde indicasse a possibilidade de acompanhamento dessas, de modo corresponsável com os serviços especializados, por Unidades de Atenção Básica, entretanto, esse processo de descentralização ainda possui vários problemas como a necessidade de uma melhor estruturação para suportar a inserção do atendimento de PVHA, desconhecimento dos profissionais de saúde sobre o manejo dessa população e a fragilidade no fluxo de atendimento dos centros de cuidado de PVHA (ZAMBENEDETTI G e SILVA RAN, 2016).

Na prática, com este novo modelo de atendimento, baseado no matriciamento da rede assistencial para as PVHA, eis que surge uma dúvida de todos que já trabalham e/ou são pacientes dos serviços especializados: os médicos atuantes na atenção básica, assim como os demais profissionais necessários para um adequado atendimento, estão prontos para realizar este tipo de assistência? (ZAMBENEDETTI G e SILVA RAN, 2016).

Nesse sentido, é de fundamental importância o conhecimento prévio do Protocolo Clínico e Diretrizes Terapêuticas (PCDT) para Manejo da Infecção pelo HIV em Adultos, assim como a facilidade em consultá-lo, para o bom atendimento de PVHA por médicos na atenção básica, contudo, existem dificuldades e contextos que prejudicam a acessibilidade do médico ao material (VIELMA L, 2013).

A dificuldade em acessar o protocolo de forma prática (disponível apenas na forma de textos impressos ou virtuais) já que em muitas unidades de saúde não se dispõe de computadores ou acesso à internet para a consulta de protocolos, é a principal realidade de locais de trabalho médico atualmente, impedindo o acesso ao material confiável e fragilizando as condutas médicas no paciente com HIV (OLIVEIRA ARF e ALENCAR MSM, 2017).

Tendo em vista este novo modelo de assistência médica a este grupo de pacientes, assim como a constante evolução e presença de aparelhos como smartphones e tablets no meio médico e visando a formação e treinamento desses profissionais, que estarão na linha de frente deste atendimento, os aplicativos em smartphones surgem como estratégia de aprendizado de informações e conhecimentos sobre 0 assunto (OLIVEIRA ARF e ALENCAR MSM, 2017). 
Diante disso, o objetivo foi desenvolver um aplicativo para dispositivos móveis com a finalidade de auxiliar as condutas durante o atendimento de PVHA de acordo com o PCDT para manejo da infecção pelo HIV em adultos.

\section{METODOLOGIA}

Para o desenvolvimento do aplicativo proposto foram utilizadas três ferramentas: ambiente Android Studio, emulador de celular e um banco de dados.

Inicialmente foi utilizada a ferramenta Android Studio na Linguagem Java como um ambiente de desenvolvimento integrado para construir softwares (aplicativos e programas) para a plataforma Android. A partir da ferramenta anterior, foi utilizado o emulador Android SDK (Kit de Desenvolvimento de Software) para simular um celular (CORDEIRO, 2012) e manejar instrumentos operacionais, contendo bibliotecas, emulador, documentação, códigos que permitiram a criação do aplicativo. Posterior a esse processo, foi usado o sistema SQLite, ferramenta de domínio publico que sustenta recursos de banco de dados permitindo o armazenamento e intercâmbio de informações para a criação informatizada do aplicativo. Foi usado, na construção do protótipo, as recomendações do Protocolo Clínico e Diretrizes Terapêuticas para Manejo da Infecção pelo HIV em Adultos (BRASIL, 2018).

\section{RESULTADOS}

O aplicativo é composto por uma tela inicial onde são visualizados seis itens, com conteúdo de acordo com o protocolo clínico e diretrizes terapêuticas para o manejo da infecção pelo HIV em adultos (Figura 1.):

1. Exames na Primeira Consulta Médica: listagem dos exames a serem solicitados na primeira consulta do paciente PVHA;

2. Iniciando a Terapia Antirretroviral (TARV): indicação do início da terapia antirretroviral e os esquemas terapêuticos indicados de acordo com seu contexto no momento da consulta (paciente sem comorbidade, gestante, mulher em idade fértil que deseja engravidar e paciente com tuberculose com ou sem gravidade clínica);

3. Profilaxias Primárias: descrição das drogas necessárias e do momento correto para a realização de profilaxias primárias, considerando a contagem de linfócitos $\mathrm{T} \mathrm{CD4}^{+}$, e resultado da Prova Cutânea Tuberculínica;

4. Quando Encaminhar ao Especialista?: lista de contextos clínicos (paciente coinfectado com hepatite $B$ ou $C$, suspeita de falha virológica, efeitos adversos com necessidade de mudança de terapia antirretroviral) ou perfil do paciente (criança, gestantes após a solicitação de exames e início da terapia antiretroviral);

5. Imunização: esquema vacinal para pacientes maiores de 13 anos infectados pelo HIV representado em uma tabela com as vacinas e seus critérios de indicação;

6. Interações Medicamentosas entre ARV e outros Medicamentos: tabela em que constam as interações medicamentosas entre os ARV ofertados no Brasil pelo Sistema Único de Saúde e as principais medicações utilizadas pelos pacientes, sendo estas organizadas segundo os sistemas de ação: cardiovascular, SNC, anti-infecciosos e outros. 
Figura 1. Área inicial do App HIV.

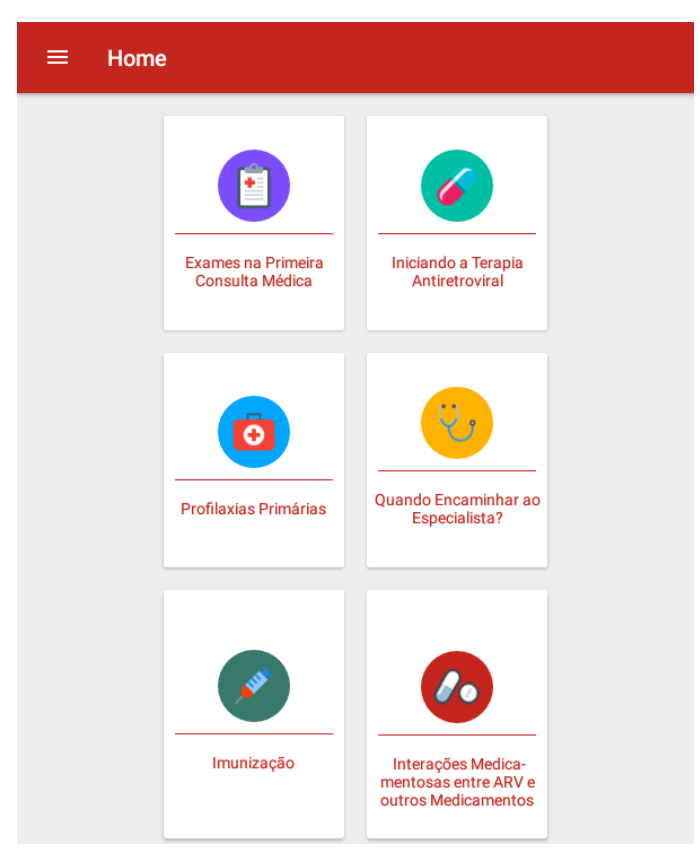

Fonte: Autores

Além disso, o aplicativo disponibiliza a visualização direta do PCDT de modo off line com a opção de realização de download (para este faz-se necessário conexão com a internet) e também a localização e contato dos serviços de assistência especializada em HIV/AIDS em Belém-Pará. Destaca-se ainda que as ferramentas do aplicativo são de execução off-line e que a conexão com a internet é necessária apenas para o download e instalação do aplicativo (Figura 2.).

Figura 2. Página de entrada do aplicativo

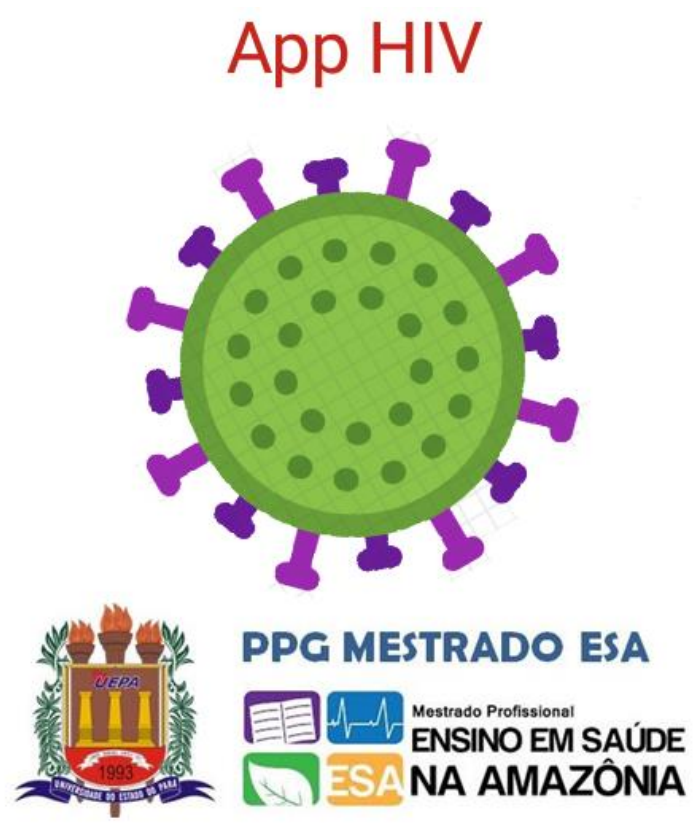

Fonte: Autores 


\section{DISCUSSÃO}

Durante a última década a Organização Mundial de Saúde (OMS) passou a considerar a infecção pelo HIV/Aids como um processo crônico de adoecimento, o que alertou para a necessidade de atenção integral das pessoas que vivem com HIV/AIDS no contexto multiprofissional com acesso a assistência dentro de seu próprio território, propondo mudanças no atual cotidiano assistencial que restringe-se aos serviços especializados, principalmente devido a sua organização e experiência dos profissionais. (PALACIO MB et al, 2014)

Desse modo, torna-se imprescindível que por meio da presença e extensão dos novos atributos que possam ser repassados a Assistência Primária à Saúde (APS), sejam identificadas as necessidades de reorganização das ações para uma atenção à saúde de qualidade e uma das formas é capacitar e treinar os profissionais que nela atuam de modo que correspondam ao novo momento que se aproxima (BATISTA KBC e GONCALVE OSJ, 2011).

Várias abordagens mostram que "a integralidade não se realiza nunca em um único serviço: integralidade é o objetivo da rede" e complementa que equidade implica no atendimento de necessidades diferenciadas da população, como por exemplo, de vínculos, de tecnologia, de condições de vida e na forma de construir autonomia para viver. (PALACIO MB, 2014). Cabe salientar que, dentre as políticas de saúde, a que mais se aproxima do princípio de "horizontalização" de acesso proposto pelo SUS (Sistema Único de Saúde) é a Política Nacional de Aids, pois o atendimento abrange os vários níveis de atenção em saúde. Entretanto, observa-se que o preparo específico para atender os usuários estaria restrito aos profissionais de centros especializados, e que tal restrição resulta na descontinuidade da assistência para as PVHA (VIELMA L, 2013; HALLAL R, 2014).

De acordo com Merces RE e Rederiro MMP (2015), em estudo realizado com uma equipe de ESF (Estratégia Saúde da Família), as pessoas que vivem com HIV/AIDS foram consideradas iguais aos demais usuários quando diz respeito aos direitos de acesso ao atendimento. No entanto, a equipe percebe o portador de maneira diferente quando o assunto são as necessidades implicadas no cuidado. (PALACIO MB, 2014). Se por um lado há um crescente quadro epidemiológico das condições crônicas, no Brasil, dentre as quais está o HIV, por outro, historicamente, os serviços de APS estavam voltados a atender os problemas agudos, interferindo negativamente na eficiência e na qualidade do acompanhamento permanente de saúde. A fragmentação na organização desses serviços pode prejudicar a coordenação do cuidado pela (NASCIMENTO L et al, 2016; GUIMARÃES MDC et al, 2017).

O Ministério da Saúde lançou uma diretriz que analisa e explica a reorganização dos modelos de atenção no manejo da infecção pelo HIV, após a avaliação do cenário nacional e experiência exitosa de alguns serviços municípios que transformara a atenção primária em saúde o foco do manejo da infecção pelo HIV/AIDS.O eixo dessa reorientação se embasa no conceito de cuidado integral e biopsicossocial, abrangendo todas as esferas do paciente (social, biológica e psicológica) visando a promoção da qualidade de vida e intervenção em fatores de risco. (SOUZA JF et al, 2015)

Para desenvolver a educação continuada de profissionais de saúde atuantes da atenção básica, foram desenvolvidos diretrizes pelo Departamento das IST, do HIV/AIDS e das Hepatites Virais montando um manejo do paciente com infecção pelo vírus HIV em todos os níveis de atenção na saúde. Esses documentos contém informações de diagnóstico, manejo clínico e tratamento, fundamentado na ética médica, sigilo, construção de ações em saúde, sendo o usuário de saúde um ator ativo desse contexto. (SOUZA JF et al, 2015).

Para o desenvolvimento de uma rede eficiente no manejo do paciente com HIV, precisou-se desenvolver passos principais como estabelecer um padrão de estratificação do risco de acordo com cada paciente, analisando sua situação clínica onde as decisões dependerão dos contextos locais, a capacidade da rede e a disponibilidade de profissionais de saúde no local; qualificação permanente dos profissionais da atenção 
básica no manejo do paciente com HIV; assegurar suporte técnico aos profissionais de saúde; disponibilizar os exames de contagem de células TCD4, contagem de carga viral do HIV e os testes rápidos de HIV; disponibilizar o acesso ao medicamento de combate ao HIV (antirretrovirais) por meio da utilização das unidades dispensadoras. (SOUZA JF et al, 2015).

Nesse sentido, como parte destas etapas, é fundamental que o médico se atualize com os novos procedimentos terapêuticos, métodos diagnósticos e condutas clínicas (inseridas no PCDT) que serão oferecidas as PVHA, contudo a preparação e reciclagem de conhecimentos do profissional médico se torna precária frente à quantidade de afazeres profissionais e pessoais. (OLIVEIRA ARF e ALENCAR MSM, 2017)

Aliado a isso existe o fato de atualmente, o PCDT em HIV/AIDS, ser disponibilizado unicamente em formato digital causando dificuldades para o seu acesso já que grande parte da rede de atenção básica não dispõe de internet e em alguns casos sequer de computadores devido, muitas vezes, estarem localizadas em áreas que sofrem com violência urbana tornando a unidade insegura para a utilização destes. Este cenário gera um contexto de dificuldade no atendimento adequado as PVHA (OLIVEIRA ARF e ALENCAR MSM, 2017)

Sobre isso, destaca-se o uso das tecnologias em celulares, por profissionais de saúde, como instrumentos de aprendizagem, reciclagem e atualização de conhecimentos. (MERCES JMR e REDERIRO MMP, 2015; ZOLFO M et al, 2010)

Com a constante evolução tecnológica e a popularização dos smartphones assim como dos tablets, os profissionais da área de saúde passaram a possuir ao seu lado um importante aliado na tomada de decisões, monitoramento, diagnóstico e prescrição de medicamentos, através da utilização de aplicativos que permitem grande auxilio durante o atendimento ao paciente sendo assim uma estratégia de aprendizado e melhoria na precisão e segurança nas condutas a serem tomadas além de levar ao profissional uma constante atualização de conhecimento. (OLIVEIRA ARF e ALENCAR MSM, 2017)

Dentre estas tecnologias, o uso dos dispositivos móveis pode ser considerado de grande importância como apoio a qualificação de profissionais devido a possibilidade de fácil acesso às informações independente do lugar e hora, além de permitirem a conexão e atualização constantes destas informações, gerando assim determinantes imprescindíveis a estes profissionais (DIAS KN, WELFER D, 2016).

Dessa forma, o aplicativo desenvolvido na pesquisa alcança objetivos favoráveis à utilização em qualquer local haja vista funcionar em ambiente off-line não necessitando de internet para manuseio, estar atualizado com as novas abordagens diagnósticas e terapêuticas do manejo do paciente com HIV/AIDS por utilizar como fonte o PCDT do Ministério da Saúde como referência em sua base de dados, ser didático em seu processamento pedagógico de informações por focar nos aspectos mais relevantes para auxiliar o médico da atenção básica, além de permitir ser utilizado em qualquer aparelho móvel (Telefone Celular ou Tablet) não precisando de material impresso ou utilização de computadores para o acesso à informação e desenvolvimento de habilidades importantes para o atendimento do paciente com HIV/AIDS.

\section{CONCLUSÃO}

O aplicativo App HIV desenvolvido pela pesquisa é benéfico em orientar o médico que atua na atenção básica com informações, direcionamento e manejo de pacientes que possuem HIV/AIDS, devendo ser difundido e utilizado pelos profissionais médicos. Além disso, é de fundamental importância que sejam desenvolvidas pesquisa científicas que estimulem a produção de aplicativos, para celulares e tablets, com informações médicas de manuais, protocolos e consensos utilizados no Brasil, facilitando o acesso a informação confiável e atualizando o conhecimento médico dos profissionais. 


\section{REFERÊNCIAS}

1. BATISTA KBC, GONCALVE OSJ. Formação dos profissionais de saúde para o SUS: significado e cuidado. Saude soc. 2011; 20(4): 884-899.

2. DIAS KN, WELFER D. Geração automática de aplicativos móveis a partir de protocolos clínicos. In XV Congresso Brasileiro de Informática em Saúde, 2016, Goiânia, 2016. Anais.

3. OLIVEIRA ARF, ALENCAR MSM. O uso de aplicativos de saúde para dispositivos móveis como fontes de informação e educação em saúde. RDBCl: Revista Digital de Biblioteconomia e Ciência da Informação, 2017; 15(1): 234-245.

4. GUIMARÃES MDC, et al. HIV/AIDS Mortality inBrazil, 2000-2015: Are therereasons for concern?. Rev Bras Epidemiol. 2017; 20(1):182-190.

5. HALLAL R, et al. O acesso universal ao tratamento antirretroviral no Brasil. Revista Tempus Actas em Saúde Pública 2010; 4:53-66.

6. MERCES JMR, REDERIRO MMP. A importância dos dispositivos móveis como estratégia para a formação e desenvolvimento de profissionais de saúde. In $22^{\circ}$ Congresso Internacional da Associação Brasileira de Educação a distância, 2015 Aguas de Lindóia. Anais.

7. NASCIMENTO L, et al. Quality of Primary Health Care for children and adolescents living with HIV. Rev. Latino-Am. Enfermagem. 2016 ;24(e270).

8. PALÁCIO MB, et al. O cuidado em HIV/Aids e a Atenção Primária em Saúde: possibilidades de integração da assistência. Psico. 2012, 43(3): 360-367.

9. SOUZA JF, et al. Avaliação de um aplicativo para auxílio à tomada de decisão de mobilizar pacientes críticos. Rev.Saúde Com. 2015; 11(1): 59-68.

10. VIELMA L. Atenção integral às pessoas que vivem com HIV/AIDS: relato de uma experiência. 28 fls. Programa De Pós-Graduação Em Residência Multiprofissional Integrada Em Sistema Público De Saúde - Universidade de Santa Maria, Santa Maria, RS, 2013.

11. ZAMBENEDETTI G, SILVA RAN. Descentralização da atenção em HIV-Aids para a atenção básica: tensões e potencialidades. Physis Revista de Saúde Coletiva. 2016; 26(3): 785-806.

12. ZOLFO M, et al. Mobile learning for HIV/AIDS healthcare worker training in resourcelimited settings. AIDS Research andTherapy. 2010, 7(35). 